\title{
Produtividade nos Serviços no Rio Grande do Sul: Dinâmica, Especialização e Desempenho*
}

\section{Dynamics, Specialization and Performance of Services Productivity in Rio Grande do Sul State}

\author{
Eduardo Pontual Ribeiro ${ }^{a}$ (i) \\ Paulo de Andrade Jacinto ${ }^{\mathrm{b}}$ if
}

\begin{abstract}
Resumo: O setor de serviços responde pela maior parte do valor adicionado da economia gaúcha e por grande parte do emprego no estado. Apesar de não ser visto como motor da economia, seu desempenho pode ser determinante para o crescimento da renda. Apresenta-se uma análise inédita da evolução da produtividade desse setor de serviços no Rio Grande do Sul, comparando-se seu patamar, evolução e estrutura com os do Brasil. A estrutura produtiva e a produtividade nos serviços no estado apresentam semelhanças com as do país. A evolução da produtividade no período é negativa, em que ganhos nominais são revertidos quando avaliados a preços constantes. Utilizando-se decomposições shift-share, conclui-se que a dinâmica agregada dos serviços no Brasil e no Rio Grande do Sul depende principalmente do comportamento intra-setorial, com tímido efeito da mudança estrutural e do bônus estrutural.
\end{abstract}

Palavras-chave: Serviços. Produtividade. Decomposição.

Abstract: The service sector has the largest share of GDP and employment in the Rio Grande do Sul economy. It may be seen as a leading sector in the economy, but its dynamics may determine income growth. We present an analysis of productivity growth in the services sector in Rio Grande do Sul, comparing its level, dynamics and structure with that of Brazil. The services sector, excluding retail and government, structure in the state is similar to the country's. Productivity actually decreased in constant prices terms, following the aggregate trend. Using shift-share decompositions, we conclude that sector productivity dynamics depended mostly on the within-sector component, with small between sector and structural bonus effects, both at Rio Grande do Sul and Brazil.

Keywords: Services. Productivity. Decomposition.

JEL Classification: E24; L8; L80.

Agradecemos o apoio de Lucas Carvalho (PIBIC CNPq) na preparação dos dados do artigo. Os autores agradecem o apoio financeiro do CNPq.

a Universidade Federal do Rio de Janeiro (UFRJ), Instituto de Economia (IE). Rio de Janeiro, Rio de Janeiro, Brasil.

b Universidade Federal do Paraná (UFPR), Departamento de Economia. Curitiba, Paraná, Brasil. 


\section{1 lntrodução}

O desempenho frustrante do crescimento da economia brasileira e do Rio Grande do Sul nos últimos anos tem direcionado esforços em entender os fatores que influenciam esse crescimento, seja pelo lado da demanda, seja pelo lado da produtividade. O recente plano Brasil Mais Produtivo (MDIC, 2016) aponta a produtividade como um dos principais fatores para a competitividade e crescimento.

A evolução da produtividade do país nos últimos anos tem sido bastante fraca, mesmo assim o crescimento da produtividade contribuiu em mais de dois terços para o crescimento do PIB per capita (DE NEGRI; CAVALCANTI, 2015). Pelos dados das contas nacionais, nota-se que o fraco desempenho da produtividade no Brasil tem se concentrado na indústria, com um desempenho positivo da agropecuária e serviços (JACINTO; RIBEIRO, 2015). No que diz respeito ao Rio Grande do Sul, a produtividade da indústria encontra-se estagnada desde 2008 (CONTRI, 2015).

O setor de serviços responde por cerca de dois terços do valor adicionado na economia e a sua importância tem sido vista na literatura como um ônus (DE NEGRI; CAVALCANTI, 2015) e uma oportunidade (ARBACHE, 2015a) para o crescimento econômico. Os aspectos negativos de uma maior importância dos serviços podem ser descritos em três dimensões, a saber: o efeito patamar, o efeito composição e o efeito derivado. No caso do efeito patamar, como a produtividade nos serviços seria, em média, menor que a indústria, a especialização nos serviços, por uma questão de compilação de dados, levaria a uma redução do crescimento da produtividade. No caso do efeito composição, nas contas nacionais o setor serviços inclui, com grande importância, o setor público, cuja mensuração de produtividade apresenta dificuldades e aproximações, sendo, a grosso modo, medido apenas pela folha salarial. Por fim, sobre o efeito derivado, a literatura acredita que os serviços possuem uma demanda induzida por outros setores produtivos (por exemplo, os serviços para empresas), sem capacidade de induzir demanda ou movimentar a economia, ou se referem a atividades pouco produtivas às pessoas como manicures, restaurantes e outros - (ARBACHE, 2015b; JACINTO; RIBEIRO, 2015), por exemplo, para esta discussão.

As oportunidades estão na realidade e nos efeitos econômicos dos serviços. Primeiro, ao contrário do senso comum, o setor de serviços pode ser bastante produtivo (JACINTO; RIBEIRO, 2015). Sua heterogeneidade é marcante, o que obscurece o papel que o setor pode ter na economia. Também ao contrário do que pode parecer a primeira vista, os efeitos multiplicadores das atividades dos serviços não são desprezíveis. Para o Rio Grande do Sul, por exemplo, Sá (2014) calcula os multiplicadores da última matriz insumo produto (MIP) disponível para o estado (2008) e observa que, nos multiplicadores de valor adicionado, as ativi- 
dades com maiores multiplicadores encontram-se no setor de serviços (mesmo desconsiderando a administração pública e aluguéis). Esse mesmo padrão é observado com os multiplicadores de emprego. Por fim, Arbache (2015b) argumenta que o canal para uma maior produtividade da indústria passa necessariamente pelo desenvolvimento do setor de serviços, coroando, assim, o protagonismo do setor em uma estratégia de desenvolvimento e competitividade.

Dada a importância do setor de serviços para a economia, surpreende a escassez de estudos disponíveis sobre esse setor. Para o Rio Grande do Sul, Alonso (2007) apresenta uma análise do setor com foco na Região Metropolitana de Porto Alegre (RMPA). Bartels e Colombo (2014) avaliam relações entre indústria e serviços com foco na atividade de transportes. Lazzari (2014), em sua análise das mudanças estruturais na economia gaúcha com base nas contas nacionais, trata do papel dos serviços com as limitações do tratamento que o setor recebe nessas estimativas (abertura em poucos setores, protagonismo da administração pública).

Com intuito de contribuir com essa escassa literatura, o presente estudo apresenta uma análise para o setor de serviços no Rio Grande do Sul. Explorando os dados regionalizados da Pesquisa Anual de Serviços (PAS) do IBGE, daremos ênfase nas atividades dos serviços explorados por empresas não estatais e, exclusive, a administração pública. A análise exclui o setor de comércio e revenda de produtos (varejo e atacado), que, nas contas nacionais, está nos serviços, mas como parte das atividades do setor terciário. A análise terá foco no indicador de produtividade, dada sua importância para o crescimento econômico, apresentando a sua evolução ao longo do tempo ao explorar a última década de dados disponíveis de forma consistente, ou seja, de 2003 a 2013.

Como em qualquer análise, e no caso específico da produtividade, os desafios deste estudo envolvem as informações estatísticas. Para uma análise setorial ao longo do tempo, há de se enfrentar a mudança da classificação setorial da CNAE 1.0 para CNAE 2.0. Por isso, iremos adotar a abordagem de Jacinto e Ribeiro (2015) como sugestão metodológica para a construção de indicadores comparáveis ao longo do tempo. A disponibilidade de informações regionalizadas na PAS também é limitada, com dados de receita, folha salarial e ocupação em 31 de dezembro do ano em questão apenas. Com isso, a medida de produtividade será receita por trabalhador. Trata-se de uma medida que não considera o consumo de matérias-primas para cálculo da produtividade.

Embora os resultados devam ser lidos com a cautela necessária devido ao indicador utilizado, o emprego de receita por trabalhador para mensurar produtividade no setor de serviços não é incomum, como, por exemplo, nos estudos de Bartelsman et al. (2015) e Foster et al. (2001), entre outros. Como benchmark para análise da produtividade dos serviços no Rio Grande do Sul, utilizaremos as infor- 
mações regionalizadas da PAS, porém agregadas para o Brasil. A metodologia é consistente e permite uma comparação mais precisa dos indicadores.

Como uma ferramenta de análise para entender a evolução da produtividade no Rio Grande do Sul, será utilizada decomposições da evolução da produtividade por permitirem gerar informações relevantes a partir de um conjunto limitado de informações e trazer uma primeira análise sobre o setor (ROCHA, 2007; RIBEIRO, 2005). Futuros trabalhos podem utilizar metodologias mais complexas a partir de outras informações disponíveis.

Os principais resultados mostram que a estrutura dos serviços no Rio Grande do Sul apresenta similaridade com a do Brasil, embora com menor peso no emprego dos serviços prestados às empresas. Quanto ao valor da produtividade, o patamar e dinâmica do estado não difere da do Brasil, com queda em valores constantes ao longo do período analisado. A heterogeneidade é significativa, com uma produtividade média de $R \$ 97.610,00$ por trabalhador, em 2013, variando de $\mathrm{R} \$ 304$ mil, em telecomunicações, a $\mathrm{R} \$ 38$ mil em manutenção e reparação. ${ }^{1}$ Por fim, observou-se que a dinâmica da produtividade deve, quase em sua totalidade, ao comportamento intrassetorial, com pequena importância das realocações estruturais.

Além dessa breve introdução, o presente estudo conta com mais quatro seções: a seção dois realiza uma descrição dos dados e aspectos metodológicos; a seção três apresenta os principais indicadores estimados com uma comparação da produtividade do Brasil e Rio Grande do Sul; a seção quatro aborda as decomposições do crescimento da produtividade realizadas; e, por fim, a quinta seção traça as considerações finais.

\section{Mensuração da Produtividade dos Serviços no Rio Grande do Sul a partir da Pesquisa Anual de Serviços: Aspectos Metodológicos}

A mensuração da produtividade em setores exige a disponibilidade de algumas informações de forma consistente ao longo do tempo. Primeiro, uma medida de produtividade, relacionando uma medida de output com medida(s) de input. Segundo, a disponibilidade de deflatores para permitir o cálculo de valores monetários (ou avaliação de quantidades heterogêneas agregadas em valor) a preços constantes. Terceiro, a disponibilidade dos dados setoriais em forma compatível ao longo do tempo, seja na definição das variáveis, seja na classificação setorial (OECD, 2001).

A nossa fonte de dados é a PAS do IBGE (vários anos). A unidade de pesquisa trata-se de empresas, em um plano amostral que envolve empresas entrevistadas

1 Em 2012, a produtividade média na indústria de transformação gaúcha era de R \$ 93.988,00 (FANTINEL, 2014). 
com certeza e empresas em uma amostra, dependendo de seu tamanho e faturamento. Essa pesquisa é a mais importante sobre serviços no país e faz parte do sistema de informações econômicas para cômputo das contas nacionais. Informações regionalizadas são apresentadas a partir das informações das unidades locais sediadas em certa unidade da federação. Se a empresa não possui unidades locais e está integralmente sediada em um estado, as informações da empresa são apresentadas nessa regionalização. O grande interesse em utilizar a PAS, em vez das contas nacionais (ou contas regionais), está em isolar, nos serviços, o efeito das atividades públicas não empresariais.

As informações da regionalização são bastante limitadas em escopo e desagregação setorial. Quanto a informações relevantes para o cálculo de produtividade, há apenas a receita bruta dos serviços mais a receita de incorporação e venda de imóveis próprios ("receita"), pessoal ocupado ("PO") em 31 de dezembro do ano em questão e salários retirados e outras remunerações ("salários"). Não há medidas de custos operacionais ou investimentos, o que impede o cálculo de medidas de valor adicionado (receita menos consumo intermediário) ou medidas de produtividade multi-insumos - como a produtividade total dos fatores (OECD, 2001). Como Foster et al. (2001) e Bartelsman et al. (2013), utilizamos receita por PO, apesar de suas limitações como medida de produtividade ao não excluir o consumo intermediário. Para os serviços, isso não deve gerar muitas distorções visto que, em geral, não há transformação de matérias-primas no processo produtivo. Por outro lado, gastos como energia não são excluídos da medida de produtividade. Em alguns setores, como transporte e alimentação, a aquisição de matérias-primas pode ser relevante.

A disponibilidade de informações regionalizadas pelo IBGE apresenta abertura setorial (divisões, grupos e classes) bastante restrita. As informações no Sistema IBGE de Recuperação Automática (Sidra) se limitam à abertura em:

1. serviços prestados às famílias, que inclui:

1.1 serviços de alojamento e alimentação;

1.2 atividades culturais, recreativas e esportivas;

1.3 serviços pessoais;

1.4 atividades de ensino continuado;

2. serviços de informação e comunicação;

3. serviços prestados às empresas;

4. transportes, serviços auxiliares aos transportes e correio, que inclui:

4.1 transporte rodoviário;

4.2 outros transportes;

4.3 armazenamento e serviços auxiliares aos transportes;

4.4 correio e outras atividades de entrega;

5. atividades imobiliárias; 
6. serviços de manutenção e reparação;

7. outras atividades de serviços.

Essa abertura pode ser considerada compatível ao longo do tempo, reconhecendo-se que houve uma mudança de classificação, da CNAE 1.0 para a CNAE 2.0 a partir da PAS 2008. Para garantir a compatibilidade dos valores nominais ao longo do tempo nos setores, utilizamos a metodologia de Jacinto e Ribeiro (2015). Aproveitando a apresentação de valores para cada setor para 2007 com as duas classificações, calculamos taxas de variação de cada medida empregada (receita, PO e salários) e utilizamos essas taxas (que são imunes à troca de classificação, isto é, utilizam tanto como ponto inicial quanto como final dados na mesma classificação setorial) para reconstruir valores a partir de um ponto base de valores. Utilizamos os valores de 2007 na classificação CNAE 2.0 como base. Maiores detalhes estão disponíveis no Apêndice A de Jacinto e Ribeiro (2015).

Por fim, faz-se necessário obter deflatores setoriais. As informações da PAS são apresentadas apenas a preços correntes. $\mathrm{O}$ uso de um deflator único para todos os subsetores é desincentivado pela OECD (2001) dado que o deflator não conseguirá reproduzir a cesta de produção dos diferentes setores. As informações das contas regionais do IBGE sofrem com mudanças metodológicas significativas em 2010. Dessa forma, optamos por utilizar os deflatores setoriais para serviços obtidos através da operação das medidas das contas nacionais trimestrais, operando índice de valor e índices a preços correntes, com alguma compatibilização de setores. Para os setores não determinados, utilizamos o indicador de "outros serviços". ${ }^{2}$ As limitações da escolha metodológica são a hipótese de mesma cesta de produção dentro de cada subsetor entre o Rio Grande do Sul e o Brasil. A obtenção de deflatores mais apropriados é certamente uma área para desenvolvimento do tema de estudo deste artigo.

Como mencionado, definimos a produtividade como receita $(Y)$ por pessoal ocupado em 31 de dezembro $(N): P_{t}=Y_{t} / N_{t}$. A medida de receita agregada pode ser escrita como a soma da receita em vários setores ou grupos $i=1, \ldots, n, Y_{t}=\sum_{i=1}^{n} Y_{i t}$, e a fração do emprego agregado no setor $i$ pode ser escrita como $w_{i t}=N_{i t} / N_{t}$. A produtividade agregada pode ser escrita, dessa forma, como a soma da produtividade em cada grupo $i$, ponderada pelo peso de cada grupo no total:

$$
P_{t}=Y_{t} / N_{t}=\Sigma_{i}\left(Y_{i t} / N_{i t}\right)\left(N_{i t} / N_{t}\right)=\Sigma_{i} P_{i t} w_{i t}
$$

Ao analisarmos a mudança da produtividade em dois períodos, temos:

$$
\Delta P_{t}=P_{t}-P_{t-1}=\Sigma_{i} P_{i t} w_{i t}-\Sigma_{i} P_{i t-1} w_{i t-1}
$$
A relação entre setores das contas nacionais trimestrais e setores da PAS para o uso do deflator
estão no Apêndice A. 
Como pode ser observado na equação 2, a variação depende das mudanças de produtividade em cada setor (desempenho setorial) e do peso de cada setor no emprego total (estrutura). Essa variação pode ser reescrita na forma de decomposição para permitir isolar a contribuição de cada elemento do cálculo de produtividade agregada, a saber, a produtividade setorial e a estrutura produtiva (pesos dos setores no pessoal ocupado). Há várias formas de fazê-lo, como descrito por Ribeiro (2005), Jacinto e Ribeiro (2015) e Rocha (2007). A mais popular usa o mesmo período como base de comparação e não impõe a ortogonalidade entre mudanças estruturais e crescimento da produtividade. Logo, a expressão segue:

$$
\Delta P_{t}=\sum_{i} \Delta P_{i t} w_{i t-1}+\Sigma_{i} \Delta w_{i t} P_{i t-l}+\Sigma_{i} \Delta P_{i t} \Delta w_{i t}
$$
(A)
(B)
(C)

na qual (A) mede a contribuição para a variação da produtividade em cada setor, mantida constante a mudança da estrutura produtiva (peso de cada setor no pessoal ocupado agregado), (B) mede a contribuição da mudança da distribuição setorial do emprego para o crescimento da produtivo, mantida a produtividade no período inicial de análise e $(C)$ mede um efeito de covariância, positivo ou negativo, entre o crescimento das variáveis que compõem a produtividade agregada. Rocha (2007) chama esse termo de bônus estrutural. O bônus estrutural indicaria um círculo virtuoso na economia, em que setores com ganho de produtividade e consequente ganho de competitividade (com possível efeito benéfico sobre os preços) conseguem fazê-lo com aumento de importância do setor na distribuição setorial do emprego.

Aqui cabe um comentário: esse tipo de decomposição, quando avaliado para empresas (como no estudo de Foster et al., 2001), tem uma interpretação natural para o bônus estrutural, visto que as empresas mais produtivas ficariam mais competitivas e conseguiriam tomar importância de empresas menos produtivas. Para uma análise setorial, o argumento de bônus estrutural via competição entre setores perde força, pois há limites para substituição entre produtos de diferentes setores. Por exemplo, embora os preços relativos possam se alterar entre serviços prestados às empresas e serviços pessoais, um eventual ganho de importância relativa de um setor em relação a outro fica limitado pelo perfil de consumo e da própria estrutura produtiva, que apresentaria baixa elasticidade substituição entre setores.

Como destacam Jacinto e Ribeiro (2015), a decomposição pode ser apresentada com variações relativas. Temos para a equação 4: 


$$
\frac{\Delta P_{t}}{P_{t-1}}=\sum \Delta P_{i t} \frac{w_{i t-1}}{P_{t-1}}+\sum \Delta w_{i t} \frac{P_{i t-1}}{P_{t-1}}+\sum\left(\Delta P_{i t}\right)\left(\Delta w_{i t}\right) \frac{1}{P_{t-1}}
$$

fazendo $g_{t}=\Delta P_{t} / P_{t-1}, g_{i t}^{W}=\Delta w_{i t} / w_{i t-1}, g_{i t}^{P}=\Delta P_{i t} / P_{i t-1}$ e $\omega_{i t-1}=\left(P_{i t-1} w_{i t-1}\right) / P_{t-1}=\left(Y_{i t-1} /\right.$ $\left.N_{i t-I}\right)\left(N_{i t-1} / N_{t-1}\right)\left[1 /\left(Y_{t-1} / N_{t-1}\right)\right]=Y_{i t-1} / Y_{t-1}$, em que $\omega$ é a parcela do setor $i$ na produção agregada, portanto a equação 4 pode ser escrita como:

$$
g_{t}=\sum g_{i t}^{P} \omega_{i t-I}+\sum g_{i t}{ }^{W} \omega_{i t-1}+\sum g_{i t}{ }^{W} g_{i t}{ }^{P} \omega_{i t-I}
$$

\section{Produtividade dos Serviços na Economia Gaúcha: Estrutura e Evolução}

A Tabela 1 apresenta a distribuição do valor agregado por setor de atividade no Rio Grande do Sul para o período de 2002 a 2013, dada a disponibilidade de dados. O ajuste ao longo do tempo para os diferentes setores dos serviços não é perfeito e pode influenciar a comparação no longo prazo. As informações dessa tabela permitem ilustrar tendências aproximadas. Verifica-se a importância do setor de serviços, que pode ter crescido ao longo do tempo, em movimento alinhado com o padrão de desenvolvimento do Brasil e de outros países. Mesmo excluindo a administração pública e os serviços de educação e saúde prestados por entes públicos e o comércio, temos uma significativa importância dos serviços, em torno de $35 \%$ em 2013. 


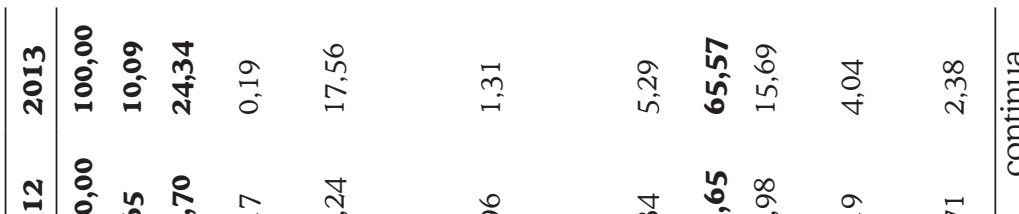

ปे

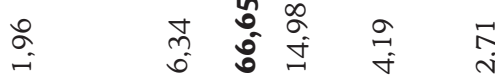

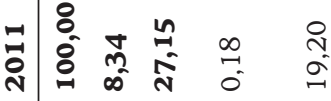

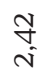

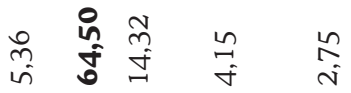

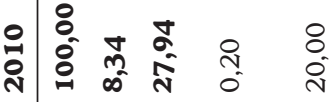

$\stackrel{\infty}{\stackrel{i}{i}}$

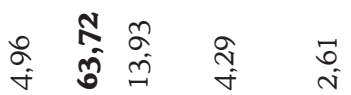

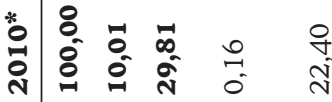

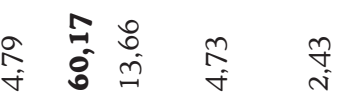

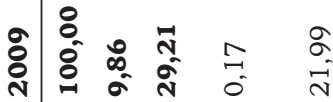

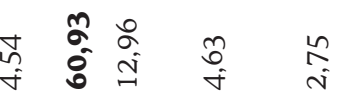

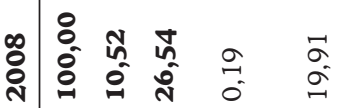

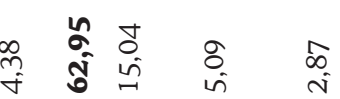

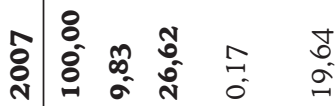

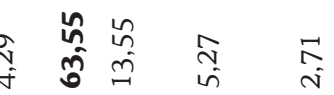

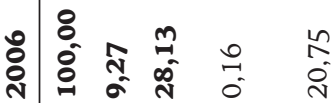

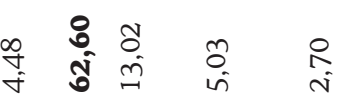

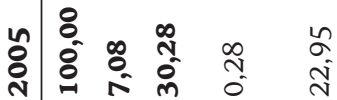

$\stackrel{\infty}{i}$

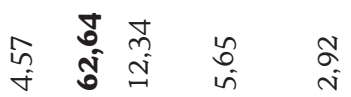

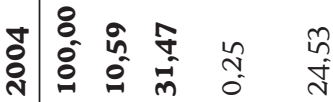

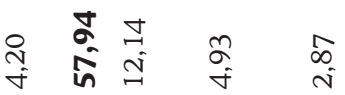

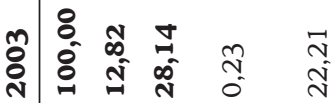

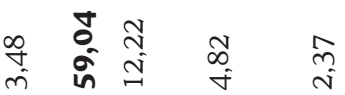

$\stackrel{\widetilde{7}}{\exists}$

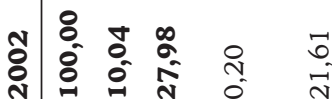

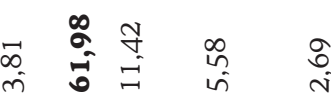

$\frac{\pi}{\mathbb{0}}$

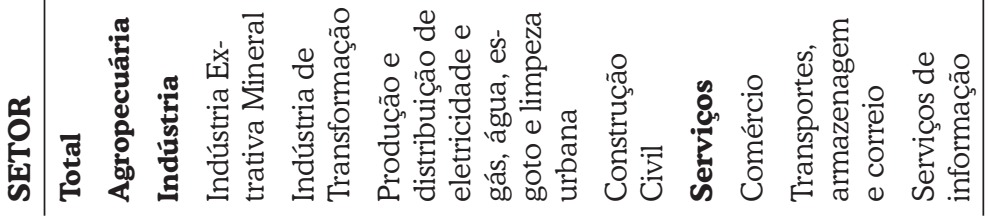




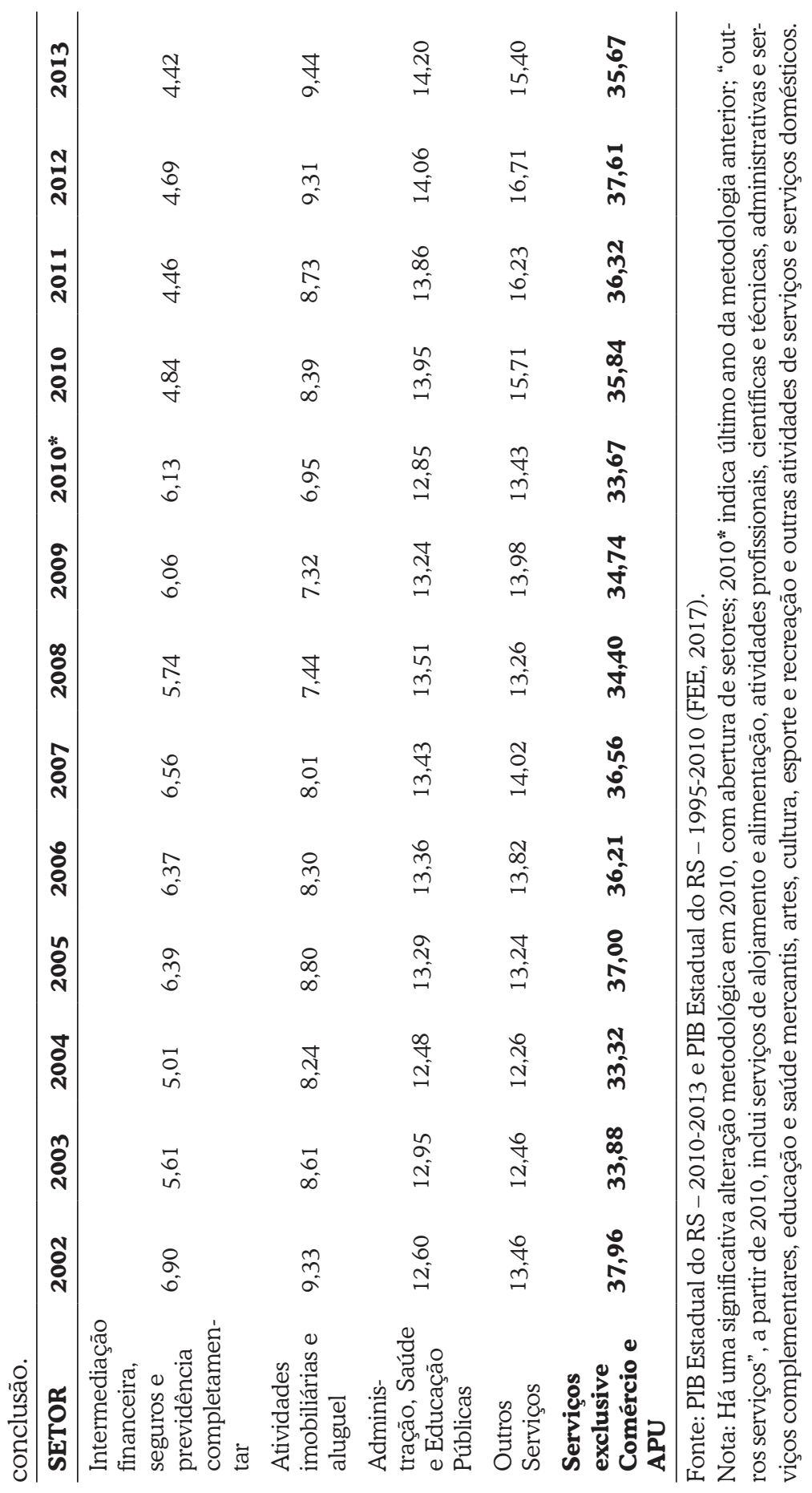


Quanto à estrutura do emprego dos setores de serviços empresariais, exclusive administração pública, como medida pela PAS, as Figuras 1 e 2 trazem a distribuição por setores e subsetores para o Rio Grande do Sul e para o Brasil. Vemos que a estrutura dos serviços no Rio Grande do Sul não difere significativamente da do Brasil. O emprego concentra-se em serviços prestados a empresas, seguido por serviços prestados às famílias e por transportes (principalmente o rodoviário). A diferença mais marcante está na maior importância de serviços prestados às famílias no Rio Grande do Sul em relação ao Brasil, com redução da importância, no estado, de serviços as empresas, apesar de mantido o ranking relativo.

Figura 1 - Distribuição do pessoal ocupado em 31 de dezembro nas atividades do setor de serviços: PAS (Rio Grande do Sul, 2003, 2007 e 2013)

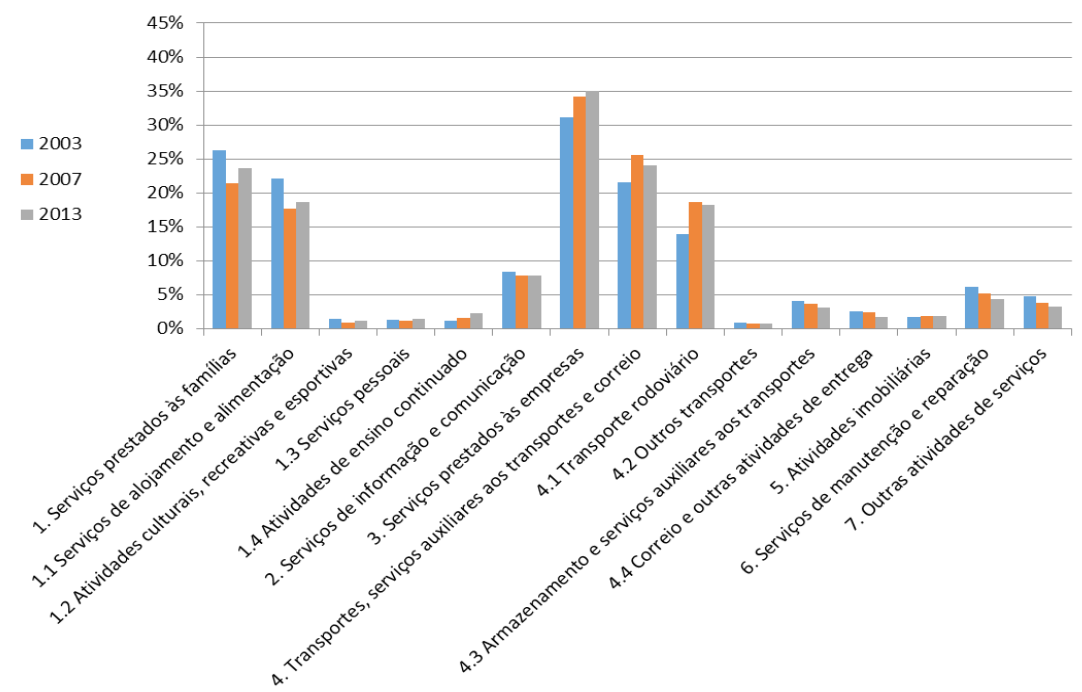

Fonte: Elaboração própria a partir de PAS/SIDRA - IBGE (IBGE, 2017).

Nota: As parcelas para os setores 1, 2, 3, 4, 5, 6 e 7 somam $100 \%$. 
Figura 2 - Distribuição do pessoal ocupado em 31 de dezembro nas atividades do setor de serviços: PAS (Brasil, 2003, 2007 e 2013)

Parcela de Ocupação por subsetor.

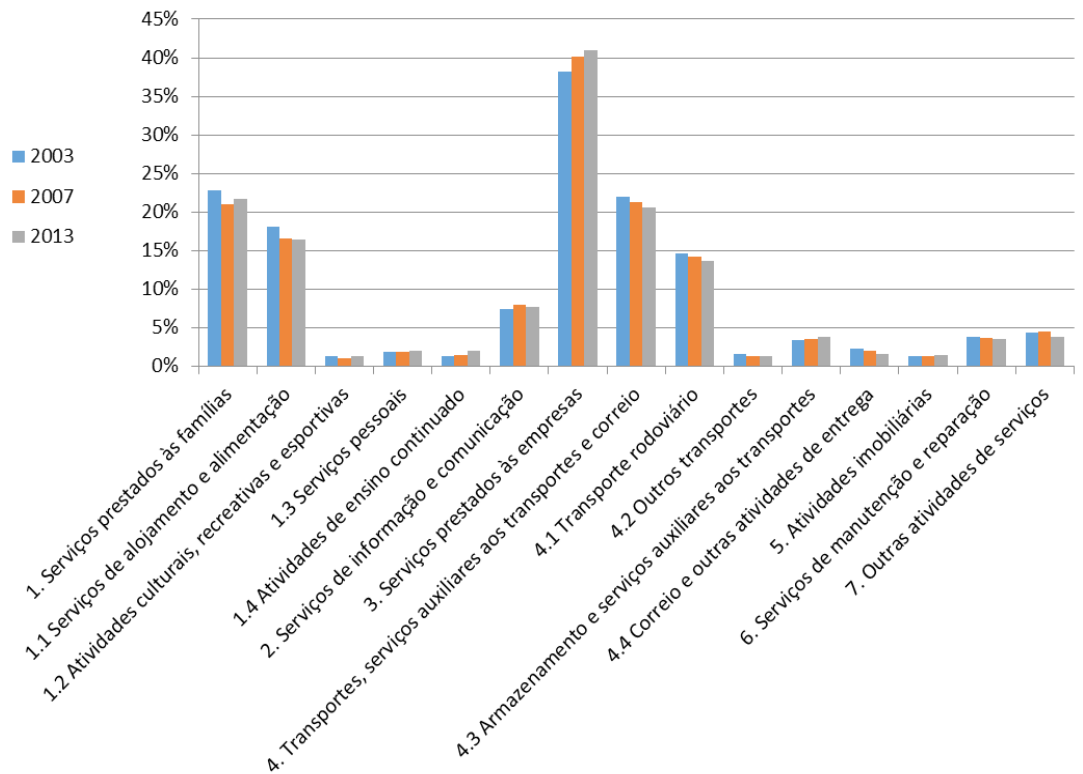

Fonte: Elaboração própria a partir de PAS/SIDRA - IBGE (IBGE, 2017).

Nota: As parcelas para os setores 1, 2, 3, 4, 5, 6 e 7 somam 100\%. 
Figura 3 - Produtividade nos serviços (em R\$): PAS (Brasil e Rio Grande do Sul, 2003-2013)

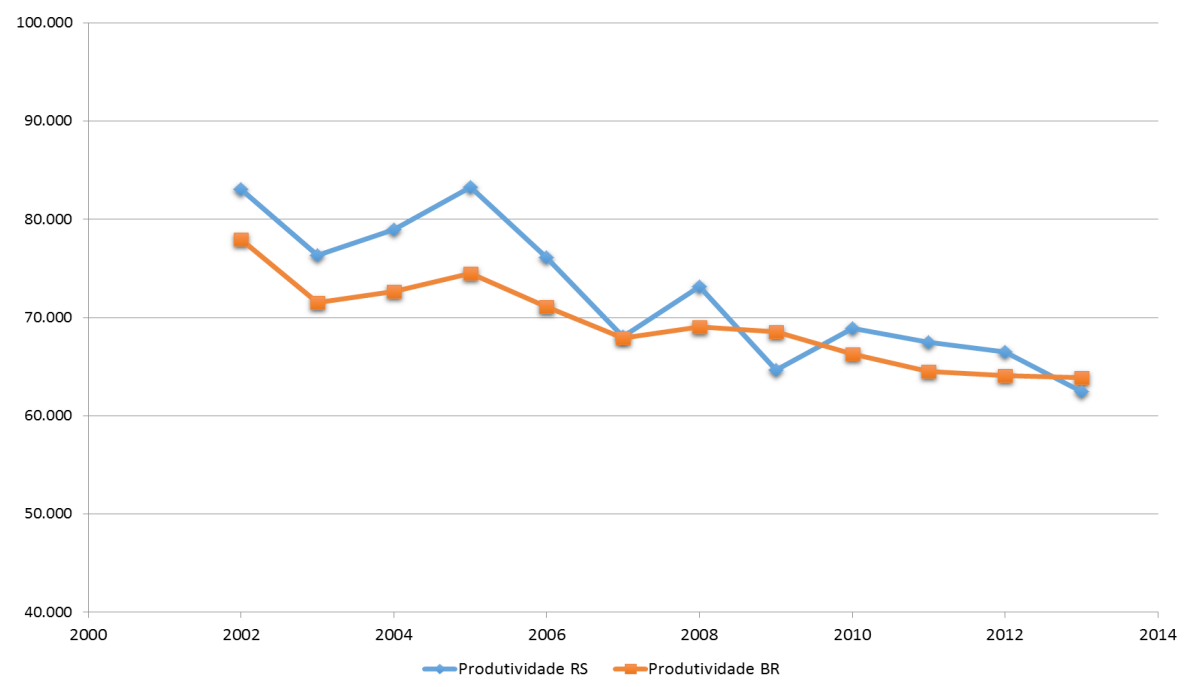

Fonte: Elaboração própria a partir de PAS/SIDRA - IBGE (IBGE, 2017).

Nota: Produtividade medida como receita bruta por pessoal ocupado em 31 de dezembro a preços de 2008 .

O patamar e dinâmica da produtividade dos serviços coletados na PAS do IBGE (IBGE, 2017) tanto para o Rio Grande do Sul quanto para o Brasil pode ser visto na Figura 3. Nota-se que os patamares são similares e as tendências muito próximas. No estado, a queda da produtividade foi mais acentuada e menos volátil (sendo que a última característica é esperada pela medida Brasil ser um conjunto maior de empresas). A preços de 2008, a produtividade, medida como receita bruta por pessoal ocupado em 2013 no Brasil e Rio Grande, estava em cerca de $\mathrm{R} \$ 63.000,00$ por trabalhador. No período de 2003 a 2013, a queda na receita por pessoal ocupado foi de $18 \%$.

A Figura 4 apresenta os componentes da produtividade agregada, a receita e o emprego, apresentados como números índice a partir de 2002. Observa-se que a queda de produtividade se deu pelo aumento mais rápido do pessoal ocupado em relação à receita em ambos os recortes regionais. Esse aumento do emprego mais rápido não se deu por expansão acelerada de setores menos produtivos ou de menor receita, visto que houve uma mudança relativa na importância dos setores muito pequena. ${ }^{3}$

3 Utilizando-se o índice de Duncan de turbulência, entre 2003 e 2013 menos de 2\% das ocupações se realocou entre setores. 
Figura 4 - Evolução do emprego e receita (a preços de 2007) no Rio Grande do Sul e Brasil (índices $2003=100$ )

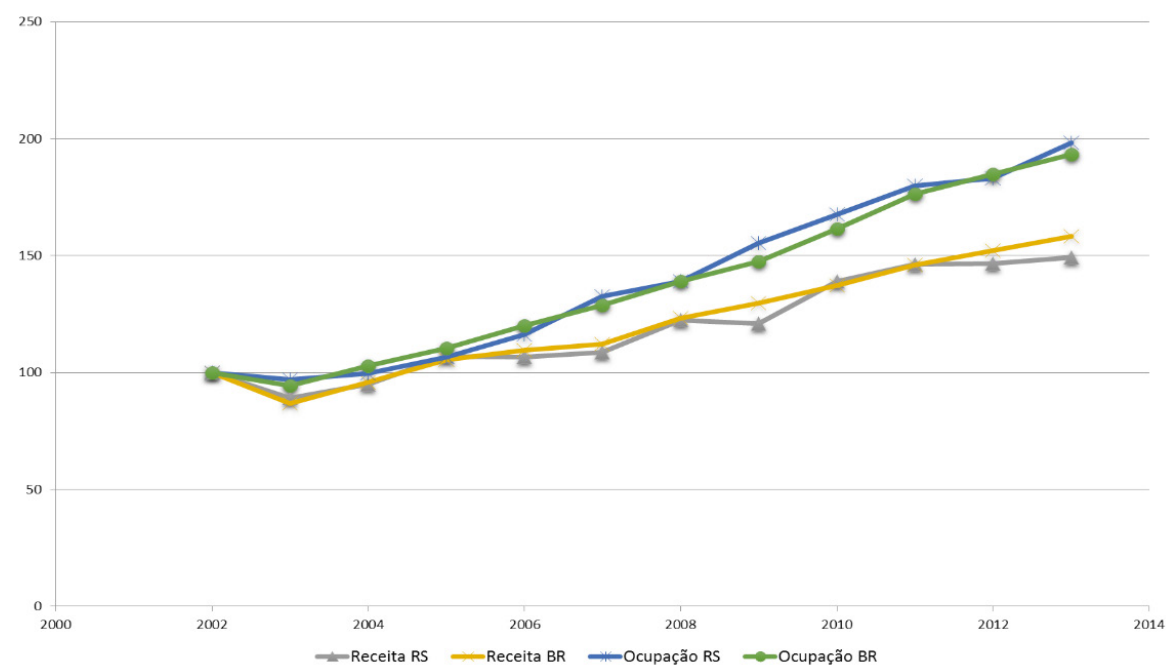

Fonte: Elaboração própria a partir de PAS/SIDRA - IBGE (IBGE, 2017).

Figura 5 - Produtividade dos serviços: Rio Grande do Sul (receita por pessoal ocupado em 31 de dezembro) em R\$ Mil (2003-2013)

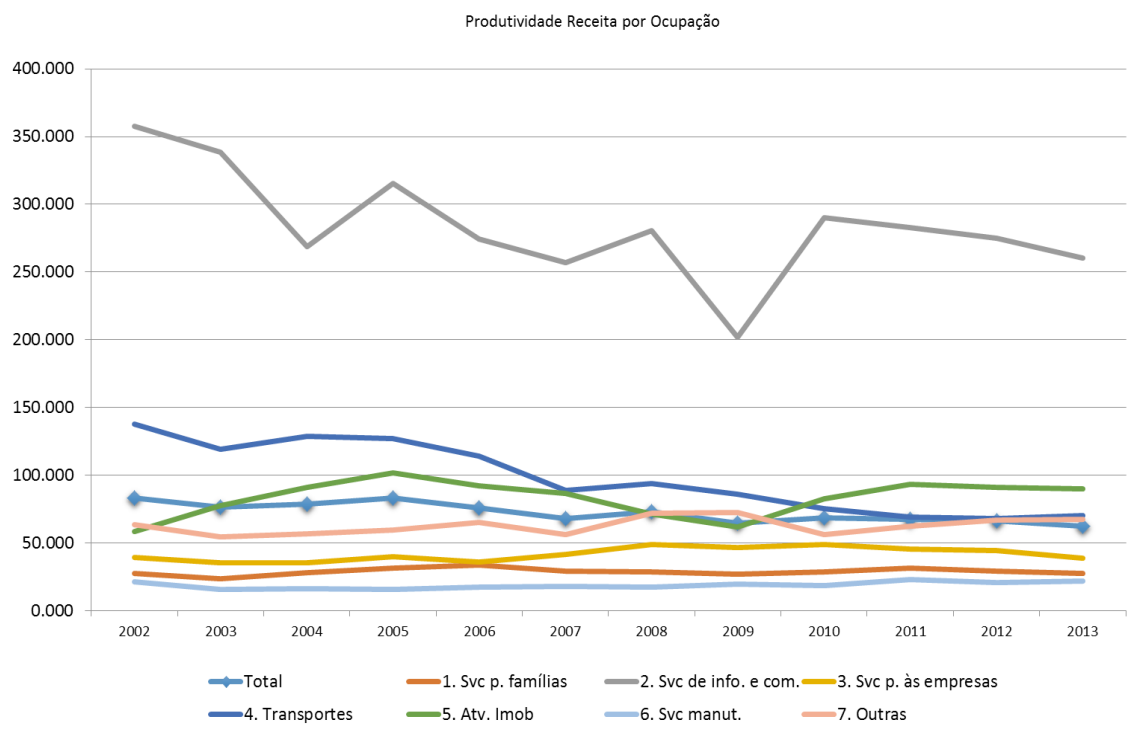

Fonte: Elaboração própria a partir de PAS/SIDRA - IBGE (IBGE, 2017).

Nota: Produtividade medida como receita bruta por pessoal ocupado em 31 de dezembro a preços de 2008. 
Figura 6 - Produtividade dos serviços: Brasil (receita por pessoal ocupado em 31 de dezembro) em R\$ Mil (2003-2013)

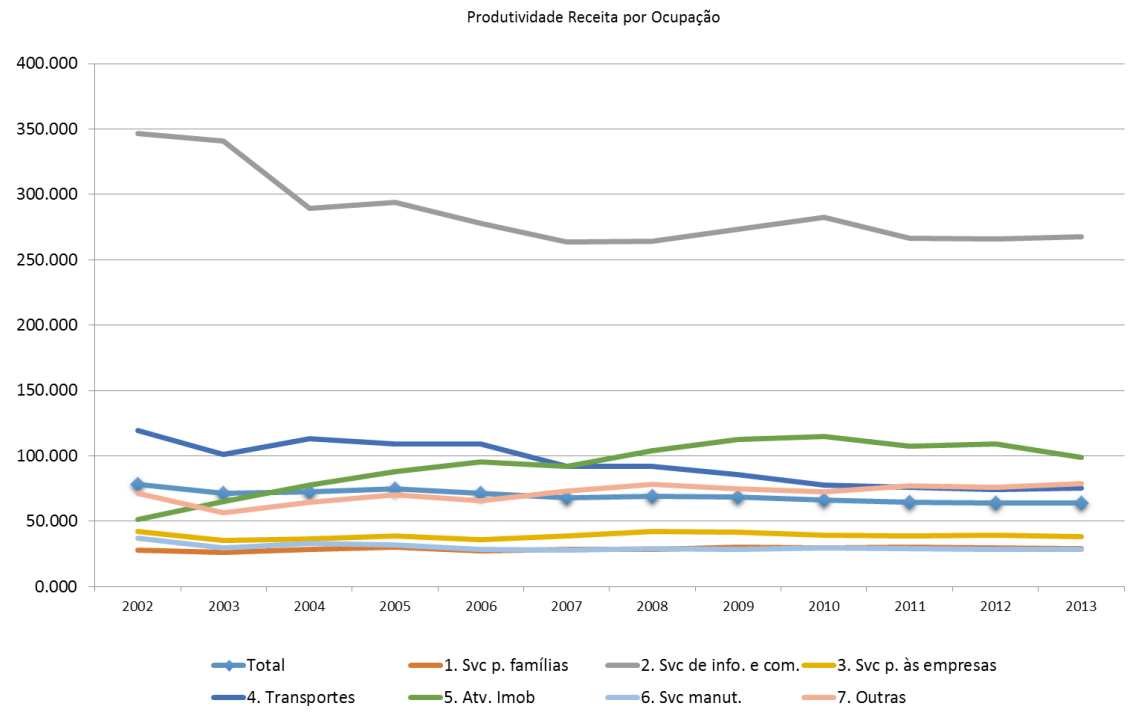

Fonte: Elaboração própria a partir de PAS/SIDRA - IBGE (IBGE, 2017).

Nota: Produtividade medida como receita bruta por pessoal ocupado em 31 de dezembro a preços de 2008.

O desempenho do Rio Grande do Sul não foi específico do estado. A Figura 6 mostra o comportamento da produtividade dos serviços para o Brasil e o padrão é bastante similar: queda significativa da produtividade do setor mais produtivo. Uma diferença entre o Brasil e o Rio Grande do Sul está no aumento da produtividade do setor de atividades imobiliárias mais forte no país do que no estado. Em geral, os padrões por setor são muito similares, não indicando uma especialização ou diferença tecnológica marcante. É interessante notar que, ao contrário dos produtos industriais, que podem ser produzidos em regiões diferentes do consumo, os serviços tendem a ser consumidos e produzidos localmente. A similaridade dos padrões de evolução de produtividade sugere uma semelhança entre regiões brasileiras no padrão tecnológico dos serviços. Forças nacionais de demanda teriam menos importância para a trajetória local dos serviços por causa da limitação da transmissão de choques ao longo do território pelo lado da demanda de produtos.

\section{Decomposição da Dinâmica da Produtividade dos Serviços}

Passamos agora para a apresentação das decomposições da evolução (queda) da produtividade no período 2003 a 2013 nos serviços. A Tabela 2 mostra que 
a quase totalidade da queda de $18 \%$ na produtividade no período se deu pelo desempenho intrassetorial. A contribuição da mudança estrutural (soma dos termos estrutural e covariância, que possuem $D w_{i t}$ ) chega a ser negativa também, mas bem menor, não respondendo por 5\% da variação acumulada. Se a produtividade intrassetorial não tivesse mudado, teríamos um leve acréscimo de produtividade, visto o componente estrutural de $1 \%$. O termo de covariância seguiu o sinal do agregado, apontando, nesse sentido, para um (limitado) bônus estrutural.

Tabela 2 - Decomposição da variação da produtividade (receita por ocupação) nos serviços no Rio Grande do Sul: PAS

\begin{tabular}{lrrrr}
\hline Periodo & \multicolumn{1}{c}{ Total } & \multicolumn{1}{c}{ Intrassetor } & Estrutural & Covariância \\
\hline $2003-2013$ & $-18.2 \%$ & $-17.3 \%$ & $1.2 \%$ & $-2.1 \%$ \\
$2003-2013$ & -13.886 & -13.245 & 0.933 & -1.573 \\
Importância relativa & & $95.4 \%$ & $-6.7 \%$ & $11.3 \%$ \\
\hline
\end{tabular}

Fonte: Elaboração própria a partir de PAS (IBGE, 2017).

Nota: Valores a preços constantes de 2007; produtividade do trabalho = receita / ocupação; para explicações sobre os termos, ver equação 5.

A Tabela 3 avalia o comportamento em dois horizontes temporais: 2003-2007 e 2007-2013. A escolha de 2007 como período intermediário para análise passou por ser um ano de consistente crescimento econômico antes da crise de 2008 e por ser um ano de mudança de classificação setorial, que pode ter influenciado a importância dos componentes na decomposição. A importância do comportamento intrassetor se mantém para explicar a dinâmica da produtividade nos subperíodos. Como diferença, temos apenas que o efeito estrutural foi maior até 2007 e de sinal inverso ao período pós-2007.

Tabela 3 - Decomposição da variação da produtividade (valor adicionado por ocupação) nos serviços no Rio Grande do Sul: PAS

\begin{tabular}{lrrrr}
\hline Período & \multicolumn{1}{c}{ Total } & Intrassetor & Estrutural & \multicolumn{1}{c}{ Covariância } \\
\hline $2002-2007$ & $-11 \%$ & $-11.6 \%$ & $2.2 \%$ & $-1.5 \%$ \\
(em R \$ de 2007) & -8.258 & -8.852 & 1.711 & -1.117 \\
Importância relativa & & $107.2 \%$ & $-20.7 \%$ & $13.5 \%$ \\
\hline $2007-2013$ & $-8.3 \%$ & $-7.4 \%$ & $-0.8 \%$ & $0.0 \%$ \\
(em R \$ de 2007) & -5.628 & -5.057 & -0.570 & -0.001 \\
Importância relativa & & $89.8 \%$ & $10.1 \%$ & $0.0 \%$ \\
\hline
\end{tabular}

Fonte: Elaboração própria a partir de PAS (IBGE, 2017).

Nota: Valores a preços constantes de 2007; produtividade do trabalho = receita / ocupação; para explicações sobre os termos, ver equação 5. 
Mais uma vez, para avaliar o padrão dos serviços no estado, comparamos com a decomposição para o Brasil como um todo. Assim como visto anteriormente para a tendência da produtividade e a similaridade de estruturas, na decomposição o padrão visto no Rio Grande do Sul se repete para o Brasil: a queda da produtividade de $10,7 \%$ observada no período foi quase que integramente explicada pelo comportamento dos setores em si, e não por mudanças na estrutura produtiva. O padrão se mantém também nos dois subperíodos, com a repetição da importância do efeito estrutural (positivo) para o período pré-2007.

Diante das limitações dos dados e das fontes dos deflatores, apresentamos análises de robustez para as decomposições em duas direções. Inicialmente, avaliamos a variação de produtividade a preços correntes, retirando o efeito dos deflatores empregados. Posteriormente, trouxemos uma medida alternativa de produtividade: como os serviços são reconhecidamente trabalho intensivo, medimos a produtividade como diferença entre a receita e a folha salarial. Isso não aproxima nossa medida de produtividade de uma medida baseada em valor adicionado por trabalhador, mas serve para entender o comportamento da receita líquida do pagamento do insumo, que teria tido aumentos significativos de custos de mão de obra.

Tabela 4 - Decomposição da variação da produtividade (valor adicionado por ocupação) nos serviços no Brasil: PAS

\begin{tabular}{lcrrr}
\hline Periodo & \multicolumn{1}{c}{ Total } & Intrassetor & \multicolumn{1}{c}{ Estrutural } & Covariância \\
\hline $2002-2013$ & $-10.7 \%$ & $-10.7 \%$ & $1.2 \%$ & $-0.2 \%$ \\
(em R \$ de 2007) & -7.618 & -7.661 & 0.846 & -0.127 \\
Importância relativa & & $100.6 \%$ & $-11.1 \%$ & $1.7 \%$ \\
\hline $2002-2007$ & $-5.1 \%$ & $-6.1 \%$ & $1.3 \%$ & $-0.3 \%$ \\
(em R \$ de 2007) & -3.625 & -4.349 & 0.927 & -0.204 \\
Importância relativa & & $120.0 \%$ & $-25.6 \%$ & $5.6 \%$ \\
\hline 2007-2013 & $-5.9 \%$ & $-4.5 \%$ & $2.0 \%$ & $-2.4 \%$ \\
(em R de 2007) & -3.993 & -3.058 & 1.342 & -1.600 \\
Importância relativa & & $76.6 \%$ & $-33.6 \%$ & $40.1 \%$ \\
\hline
\end{tabular}

Fonte: Elaboração própria a partir de PAS (IBGE, 2017).

Nota: Valores a preços constantes de 2007; produtividade do trabalho = receita / ocupação; para explicações sobre os termos, ver equação 5. 
Tabela 5 - Decomposição da variação da produtividade (receita por ocupação) nos serviços no Rio Grande do Sul: PAS

\begin{tabular}{lrrrr}
\hline Período & \multicolumn{1}{c}{ Total } & Intrassetor & Estrutural & Covariância \\
\hline $2002-2013$ & $77.1 \%$ & $77.5 \%$ & $1.2 \%$ & $-1.6 \%$ \\
(em R\$ correntes) & 42.498 & 42.721 & 0.656 & -0.880 \\
Importância relativa & & $101 \%$ & $2 \%$ & $-2 \%$ \\
\hline $2002-2007$ & $23.5 \%$ & $22.5 \%$ & $2.2 \%$ & $-1.1 \%$ \\
(em R $\$$ correntes) & 12.977 & 12.383 & 1.201 & -0.607 \\
Importância relativa & & $95 \%$ & $9 \%$ & $-5 \%$ \\
\hline 2007-2013 & $43.4 \%$ & $45.1 \%$ & $-0.8 \%$ & $-0.9 \%$ \\
(em R\$ correntes) & 29.521 & 30.701 & -0.570 & -0.609 \\
Importância relativa & & $104 \%$ & $-2 \%$ & $-2 \%$ \\
\hline
\end{tabular}

Fonte: Elaboração própria a partir de PAS (IBGE, 2017).

Nota: Valores a preços correntes; produtividade do trabalho = receita / ocupação; para explicações sobre os termos, ver equação 5 .

A Tabela 5 mostra como o preço dos serviços cresceu durante o período de 2003 a 2013. Sem o deflator, estimou-se um aumento de 77\% na receita por trabalhador nos serviços. Por outro lado, mas uma vez o padrão de evolução da produtividade seguiu o descrito anteriormente: uma pequena contribuição estrutural e uma grande importância do comportamento intra-setorial. Os resultados para o Brasil estão disponíveis com os autores e são omitidos para poupar espaço, dada a repetição do padrão da medida de produtividade com receita deflacionada. Ao olhar nos subperíodos, é possível inferir que os preços dos serviços tiveram aceleração mais forte pós-2007.

Tabela 6 - Decomposição da variação da produtividade ((receita - folha salarial)/ ocupação) nos serviços no Rio Grande do Sul: PAS

\begin{tabular}{lrrrr}
\hline Período & Total & Intrassetor & Estrutural & Covariância \\
\hline $2002-2013$ & $-17.0 \%$ & $-16.2 \%$ & $0.9 \%$ & $-1.8 \%$ \\
(em R \$ de 2007) & -11.768 & -11.201 & 0.651 & -1.219 \\
Importância relativa & & $95 \%$ & $-6 \%$ & $10 \%$ \\
\hline $2002-2007$ & $-9.5 \%$ & $-10.2 \%$ & $1.8 \%$ & $-1.1 \%$ \\
(em R \$ de 2007) & -6.588 & -7.052 & 1.227 & -0.764 \\
Importância relativa & & $60 \%$ & $-17 \%$ & $12 \%$ \\
\hline $2007-2013$ & $-9.1 \%$ & $-8.3 \%$ & $-0.8 \%$ & $0.0 \%$ \\
(em R $\$$ de 2007) & -5.180 & -4.705 & -0.450 & -0.025 \\
Importância relativa & & $91 \%$ & $9 \%$ & $0 \%$ \\
\hline
\end{tabular}

Fonte: Elaboração própria a partir de PAS IBGE, 2017).

Nota: Valores a preços constantes de 2007; produtividade do trabalho $=$ (receita - folha salarial) / ocupação; para explicações sobre os termos, ver equação 5.

Concluindo a análise, a Tabela 6 também aponta o mesmo padrão de evolução da produtividade determinada pelo comportamento intrassetorial. Também 
disponível com os autores, a respectiva Tabela 6 para o Brasil mostra resultados similares qualitativa e, inclusive, quantitativamente em relação ao visto nas tabelas anteriores.

\section{Considerações Finais}

O setor de serviços aparece em destaque na estrutura da geração de valor adicionado e emprego no Rio Grande do Sul e no Brasil. Exclusive a administração pública e os serviços prestados por esse ente e o comércio, as atividades de serviços propriamente ditos respondem por cerca de um terço do valor adicionado na economia gaúcha, em 2013, a partir de dados da Fundação de Economia e Estatística. A importância dessas atividades contrasta com a limitada literatura no estado sobre seu comportamento e desempenho. Ao mesmo tempo, alguns autores, como Arbache (2015a), têm sugerido que uma estratégia sustentável de crescimento econômico necessita passar pelo aumento de produtividade e competitividade dos serviços, inclusive como forma de aumentar a produtividade da indústria. O objetivo deste artigo foi trazer alguns resultados sobre o setor de serviços, como mensurado na PAS do IBGE.

A literatura nacional e internacional vistas no texto destacam a heterogeneidade marcante do setor de serviços prestados por empresas. São incluídas atividades desde comunicações e informação, setor capital intensivo, com significativas economias de escalas e com alta produtividade, até serviços de manutenção e reparação, trabalho intensivo e de baixa produtividade.

O uso de dados da Pesquisa Anual de Serviços do IBGE (IBGE, 2017), indo além das contas regionais, se faz recomendado pela possibilidade de uma análise mais detalhada (ao longo do tempo) e uma divisão setorial mais ampla dos que as contas regionais (pelo menos até 2010). O uso dos dados da PAS, todavia, exige o tratamento de possíveis mudanças de patamar em 2007 com a mudança de classificação setorial e a escolha dos deflatores.

Superados esses desafios, verificamos que a produtividade dos serviços está em queda, devido a um crescimento dos preços superior ao volume de negócios. Essa queda ocorre de forma similar em magnitude no Rio Grande do Sul e no Brasil. A estrutura das atividades de serviços e a produtividade em si no estado é muito similar à do Brasil, apontando uma similaridade tecnológica entre produtores locais e em outras unidades da federação.

Por fim, como primeiro esforço para entender a evolução negativa da produtividade nos serviços gaúchos, apresentamos decomposições do crescimento da produtividade agregada, seja a regional ou a nacional. Nessas decomposições, foi possível concluir sobre a significância dos efeitos intrassetoriais e o papel coadjuvante de realocação de mão de obra entre atividades dos serviços. Quase todo 
o montante de queda da produtividade a preços constantes de 2007 se deve à variabilidade de produtividade intrassetorial e menos uma mudança na estrutura dos serviços. Há uma evidência muito tênue, pela importância relativa dessa teoria do bônus estrutural, da relevância do bônus estrutural para o desempenho da produtividade.

Esse resultado é corroborado por uma extensa análise de robustez. Seja utilizando dados não deflacionados (para isolar o efeito da escolha dos deflatores), seja utilizando uma medida alternativa de produtividade (receita menos folha de pagamento, por trabalhador), o protagonismo no comportamento intrassetorial dos serviços se mantém. Ao mesmo tempo, o padrão do Rio Grande do Sul surge de forma similar nas decomposições para o Brasil. O efeito de mudança de estrutura tende a ser de importância secundária. Já o chamado bônus estrutural foi verificado em seu sinal coerente com a evolução agrada, mas ainda com papel bastante limitado para descrever a trajetória da produtividade dos serviços.

A análise realizada aqui mostra a importância de se valorizar o desempenho do setor de serviços para se sustentar o crescimento econômico. Revertendo-se a trajetória de evolução da produtividade dos serviços, o setor pode contribuir positivamente para o crescimento de forma direta, gerando valor agregado, como de forma indireta, melhorando os serviços prestados a empresas (inclusive telecomunicações e transporte), o que evitaria a chamada "doença de custos" de Baumol.

Por fim, vale notar que a análise setorial pode ser complementada por uma análise nas empresas, utilizando-se microdados. Um grande benefício está na revelação do papel da dinâmica das empresas (entrada e saída) para a compreensão da evolução da produtividade do setor. Isso parece ser um tema relevante para futuras pesquisas.

\section{Referências}

ALONSO, J. A. F. A economia dos serviços na região metropolitana de Porto Alegre (RMPA): uma primeira leitura. Textos para Discussão FEE, n. 3, 2007.

ARBACHE, J. The contribution of services to manufacturing competitiveness in Brazil. In: MULDER, N. et. al.(ed.) Innovation and internationalization of services in Latin America, Santiago: COLEF-ECLAC-UNCTAD, 2015a.

ARBACHE, J. Produtividade no setor de serviços. In: DE NEGRI, F.;CAVALCANTE, L. (org.). R. Produtividade no Brasil: desempenho e determinantes, Brasília: IPEA, 2015b. v. 2.

BARTELS, M.; COLOMBO, J. Interdependência entre a indústria de transformação e os serviços de transporte: evidências para o Rio Grande do Sul e para o Brasil. In: ENCONTRO DE ECONOMIA GAÚCHA, 7., 2014, Porto Alegre. Anais eletrônicos [...]. Porto Alegre: FEE, 2014. 
BARTELSMAN, E. et al. Cross-country differences in productivity: the role of allocation and selection. American Economic Review, v. 103, n. 1, p. 305-334, 2013.

CONTRI, A. L. Produção, salário, emprego e produtividade na indústria do RS. Carta de Conjuntura FEE, a. 24, n. 5, 2015.

DE NEGRI, F.; CAVALCANTE, L. R. Produtividade no Brasil: desempenho e determinantes. v. 2, Brasília: IPEA, 2015

FANTINEL, V. D. Evolução e níveis de produtividade na indústria de transformação gaúcha. Carta de Conjuntura FEE, ano 23, n. 10, 2014.

FEE. Série histórica. Porto Alegre: FEE, 2017. Disponível em: https://www.fee.rs.gov.br/ indicadores/pib-rs/estadual/serie-historica/. Acesso em: 15 jan. 2019.

FOSTER, L. et al. Aggregate productivity growth: lessons from microeconomic evidence. In: HULTEN, C. et al. (ed.). New developments in productivity analysis. New York: NBER, 2001.

IBGE. Pesquisa anual de serviços - PAS. [S.1.]: IBGE, 2017. Disponível em: https://sidra.ibge. gov.br/pesquisa/pas/tabelas. Acesso em: 15 jan. 2019.

JACINTO, P. A.; RIBEIRO, E. P. Crescimento da produtividade no setor de serviços e da indústria no Brasil: dinâmica e heterogeneidade. Economia Aplicada, v. 19, n. 3, p. 401-427, 2015.

LAZZARI, M. R. A economia gaúcha de 1947 a 2013: uma análise panorâmica. In: PICHLER, W. A. et al. (org.). Panorama socioeconômico e perspectivas para a economia gaúcha. Porto Alegre: FEE, 2014.

MINISTÉRIO DO DESENVOLVIMENTO, INDÚSTRIA E COMÉRCIO - MDIC. Plano Brasil mais produtivo. Brasília, 2016. Disponível em: http://www.mdic.gov.br/index.php/ competitividade-industrial/brasil-mais-produtivo. Acesso em: 15 jan. 2019.

OECD. Measurement of aggregate and industry-level productivity growth. In: OECD. Measuring productivity, Paris: OECD Publishing, 2001. Disponível em: http://dx.doi. org/10.1787/9789264194519-en. Acesso em: 21 ago. 2019.

RIBEIRO, E. P. Produtividade e emprego na indústria do RS, 1996- 2000: especialização ou desempenhos setorial? Indicadores Econômicos FEE, v. 33, n. 2, p. 161-174, 2005.

ROCHA, F. Composição do crescimento dos serviços na economia brasileira: uma análise da matriz insumo-produto (1985-1992). Econômica, v. 1, n. 2, p. 5-21, 1999.

ROCHA, F. Produtividade do trabalho e mudança estrutural nas indústrias brasileiras extrativa e de transformação, 1970-2001. Revista de Economia Política, v.27, n. 2, p. 221-241, 2007

SÁ, R. Matriz de insumo-produto do Rio Grande do Sul - 2008: hipóteses, interpretações e principais resultados In: PICHLER, W. A. et al. (org.). Panorama socioeconômico e perspectivas para a economia gaúcha. Porto Alegre: FEE, 2014. 


\section{Apêndice A}

Quadro 1 - Relação de deflatores utilizados em subsetores

\begin{tabular}{|c|c|}
\hline Setor PAS & $\begin{array}{c}\text { Setor Contas Nacionais } \\
\text { Trimestrais }\end{array}$ \\
\hline 1. Serviços prestados às famílias & Índice de preços, serviços, outros. \\
\hline 1.1 Serviços de alojamento e alimentação & Índice de preços, serviços, outros. \\
\hline 1.2 Atividades culturais, recreativas e esportivas & Índice de preços, serviços, outros. \\
\hline 1.3 Serviços pessoais & Índice de preços, serviços, outros. \\
\hline 1.4 Atividades de ensino continuado & Índice de preços, serviços, outros. \\
\hline 2. Serviços de informação e comunicação & $\begin{array}{l}\text { Índice de prelos, serviços, } \\
\text { informações. }\end{array}$ \\
\hline 3. Serviços prestados às empresas & Índice de preços, serviços, outros. \\
\hline $\begin{array}{l}\text { 4. Transportes, serviços auxiliares aos transportes } \\
\text { e correios }\end{array}$ & $\begin{array}{l}\text { Índice de preços, serviços, } \\
\text { transportes. }\end{array}$ \\
\hline 4.1 Transporte rodoviário & $\begin{array}{l}\text { Índice de preços, serviços, } \\
\text { transportes. }\end{array}$ \\
\hline 4.2 Outros transportes & $\begin{array}{l}\text { Índice de preços, serviços, } \\
\text { transportes. }\end{array}$ \\
\hline $\begin{array}{l}\text { 4.3 Armazenamento e serviços auxiliares aos } \\
\text { transportes }\end{array}$ & $\begin{array}{l}\text { Índice de preços, serviços, } \\
\text { transportes. }\end{array}$ \\
\hline 4.4 Correio e outras atividades de entrega & $\begin{array}{l}\text { Índice de preços, serviços, } \\
\text { transportes. }\end{array}$ \\
\hline 5. Atividades imobiliárias & Índice de preços, serviços, outros. \\
\hline 6. Serviço de manutenção e reparação & Índice de preços, serviços, outros. \\
\hline 7. Outras atividades de serviços & Índice de preços, serviços, outros. \\
\hline
\end{tabular}

Fonte: Elaboração própria.

Recebido em: 23/02/2017. Aceito em: 26/10/2017. 